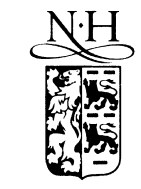

ELSEVIER

\title{
Multi-electron processes in atomic collisions - theory
}

\author{
L. Nagy ${ }^{1}$ \\ Faculty of Physics, Babeş-Bolyai University, str. Kogălniceanu nr. 1, 3400 Cluj, Romania
}

\begin{abstract}
For the theoretical description of the multi-electron processes induced by charged particle impact we use a relatively simple perturbative method. The second-order approximation is valid for protons and antiprotons of moderate and high velocities. Electron correlation is taken into account by the use of configuration-interaction wavefunctions. The agreement with the available experimental data for some two-electron processes in the helium depends on the quality of the wavefunctions used. (c) 1999 Elsevier Science B.V. All rights reserved.
\end{abstract}

PACS: $34.50 . \mathrm{Fa} ; 34.10 .+\mathrm{x}$

Keywords: Multi-electron transitions; Double ionization; Ionization-excitation; Double excitation; Helium atom

\section{Introduction}

The theoretical description of the multi-electron processes induced by charged particle impact is far from routine, even for the simplest two-electron processes in the helium target, such as double ionization, ionization-excitation or double excitation. Typically the different theoretical approximations do not agree with each other, and only a few theoretical results are in reasonable agreement with the experimental data.

It is clear, that for the description of the multielectron processes the independent-electron approximation is not valid, and special interest has to be given to the electron correlations [1]. Correlation effects are essential in the calculation of the

\footnotetext{
${ }^{1}$ E-mail: lnagy@atom.ubbcluj.ro
}

cross sections at high impact velocities, where the first-order term dominates, and the transition of the second electron is caused by electron-electron interaction. Correlation is responsible for the experimentally observed effect, that in a large velocity range cross sections for negative projectiles are different (usually higher) relative to the cross sections obtained with the same velocity positive projectiles.

Experimentally the most studied two-electron process is the double ionization of the helium [2-9]. There have been elaborated some qualitative models in explaining the velocity dependence of the cross sections, and mostly the fact, that cross sections for antiproton projectiles are significantly higher, than those obtained with proton projectiles [10-12].

The most elaborated and successful theoretical description of the double ionization is that of Reading and Ford [13]. Their forced impulse 
method divides the collision time into sequential short segments such that an impulse approximation is forced to be valid. Electron correlation is taken into account at the end of each segment, but electrons propagate independently during each segment. Practically the authors use two timesegments, and the correlation is taken into account three times during the collision. In order to describe correlation they have to solve a large set of coupled equations. Their first results [13] were only in qualitative agreement with the experiment, but later Ford and Reading have performed the calculations with an enlarged basis set, obtaining very good agreement with the experimental data [14] above $300 \mathrm{keV} / \mathrm{amu}$ projectile energy. Recently, the same group has proved, that using more time intervals, and taking into account the electron correlations several times during the collision, they can obtain good results for lower projectile energies, too [15].

Other theoretical approaches, like the dynamical classical trajectory Monte Carlo calculations of Meng et al. [16] and the independent event model of Marshall et al. [17] does not lead to cross sections consistent with the results of Ford and Reading [14] or the experimental data. The recent two-coupled-channel plane wave Born approximation calculations of Das and Malik [18] are in reasonable agreement with the experimental data, but cross sections for high energies (especially for single ionization) have a peculiar behavior, e.g. at $50 \mathrm{MeV} / \mathrm{amu}$ projectile energy, where the first Born approximation should be valid (leading to the same cross sections for protons and antiprotons), their cross sections for antiprotons are five times larger than for protons.

Regarding the other interesting two-electron processes, the situation is worse. For the ionization-excitation of the helium, calculations have been made by Rudge [19], Raeker et al. [20], Franz and Altick [21], Nagy et al. [22], Sidorovich [23]. The results of these calculations do not agree with each other, and all underestimate the experimental data [24-28]. Excepting our previous work [22] for the excitation of the $2 p$ state, and Sidorovich [23] for the excitation of the 2 s state, nobody has made an attempt to compare theoretical cross sections for same velocity positive and negative projectiles, the other calculations being only for electrons. Experimental data show the same interesting feature as for the double ionization: cross sections for electrons are obtained to be higher than for equivelocity proton projectiles in a wide velocity range.

The experimental measurement of the cross sections is more complicated for the double excitation of the helium. The difficulty of the problem arises from the nature of the doubly excited states of the helium atom, which all are autoionizing states, their energy lying above the single ionization limit. Under these conditions information about the population of the doubly excited states can be obtained by the analysis of the energy spectra of the ejected electron, the different autoionizing states appearing as resonances. The theoretical interpretation of these experimental spectra is difficult, because of the interference of the direct and resonant ionization processes, and the three-body Coulomb interaction in the final state between the scattered projectile, ejected electron and the residual ion [29].

As for the calculation of the double excitation cross section, basically this should represent a simpler case. Correlation effects in the initial and final discrete states can be taken into account more easily than for the continuum states. Different coupled channel and perturbative calculations have been performed to obtain double excitation cross sections [30-34]. In spite of the relative simplicity of the process when neglecting the interference with direct ionization, the results of these calculations did not agree with each other. In order to clarify the problem, one should compare these results to the experimental data. But the extraction of the cross sections from the experimental spectra is difficult, and needs not only a careful analysis of the involved phenomena, but a good energy resolution of the spectrometer in order to separate the peaks associated with different doubly excited states and to obtain information about the structure of these peaks. First Pedersen and Hvelplund [35], then Giese et al. [36] have reported experimental double excitation cross sections for high velocity projectiles. But because of the insufficient energy resolution of their spectrometer and the lack of measurements for backward ejection angles, the results, as stated by the 
authors, are not accurate enough. These experimental data have not been able to really test the theoretical calculations.

Very recently, the complete theoretical description of the resonant ionization processes at intermediate projectile energies $(100 \mathrm{keV} / \mathrm{amu})$ performed by Godunov et al. [29] made possible for Moretto-Capelle et al. [37] to extract double excitation cross sections from their experimental spectra of the ejected electron, obtained with a high-resolution spectrometer. These cross sections not only complete the experimental data of Giese et al. [36] to lower energies, but are stated to be more exact, making sense for the comparison of different theoretical results with the experiment.

As one can see, many aspects of the multielectron transitions are not yet clarified, and only some of the experimental data could be reproduced theoretically, so further work is needed. In the present paper we describe our impact-parameter perturbative method for the calculation of the cross sections of different two-electron transitions. The applied second-order approximation is suitable for proton and antiproton projectiles with energies above $50 \mathrm{keV} / \mathrm{amu}$. Electron correlation is taken into account by the use of configurationinteraction (CI) wavefunctions. Results are presented for the ionization-excitation and the double excitation of the helium.

\section{Theory}

We have used the impact parameter method for the description of the collision process. This approximation is valid only for fast collisions, if the associated wavelength of the projectile is less than the atomic dimension, and the energy transfer to the electrons is negligible compare to the energy of the projectile. Under these conditions the projectile can be considered as moving on a classical straight-line trajectory with constant velocity.

The cross section for a given process can be obtained by integrating the transition probability (the square of the modulus of the probability amplitude) over the impact parameter

$$
\sigma=\int \mathrm{d}^{2} \mathbf{B}|a(B)|^{2} .
$$

We calculate the probability amplitude by timedependent perturbation theory, as described in Ref. [38], where the perturbation is taken to be the sum of the projectile-electron interactions. For a two-electron system $\left(V_{1}(t)\right.$ and $V_{2}(t)$ being the interaction potentials of the two electrons with the projectile), the first and the second-order amplitude can be written as

$$
a^{(1)}=-i \int_{-\infty}^{+\infty} \mathrm{d} t \mathrm{e}^{\mathrm{i}\left(E_{f}-E_{i}\right) t}\left\langle f\left|\left[V_{1}(t)+V_{2}(t)\right]\right| i\right\rangle
$$

and

$$
\begin{aligned}
a^{(2)}= & -\sum_{k} \int_{-\infty}^{+\infty} \mathrm{d} t \mathrm{e}^{\mathrm{i}\left(E_{f}-E_{k}\right) t}\left\langle f\left|\left[V_{1}(t)+V_{2}(t)\right]\right| k\right\rangle \\
& \times \int_{-\infty}^{t} \mathrm{~d} t^{\prime} \mathrm{e}^{\mathrm{i}\left(E_{k}-E_{i}\right) t^{\prime}}\left\langle k\left|\left[V_{1}\left(t^{\prime}\right)+V_{2}\left(t^{\prime}\right)\right]\right| i\right\rangle .
\end{aligned}
$$

Here $|i\rangle,|f\rangle$ and $|k\rangle$ stand for the initial, final and the intermediate state of the electron system with the energies $E_{i}, E_{f}$ and $E_{k}$, respectively. The above expressions contain electron correlations through the wavefunctions in the initial, final and intermediate states. As described in Ref. [39], we neglect in further calculations the terms in $\left(V_{1}\right)^{2}$ and $\left(V_{2}\right)^{2}$.

The two-electron wavefunctions $|i\rangle,|f\rangle$ and $|k\rangle$ cannot be calculated exactly. A good approximation can be reached by the use of configurationinteraction (CI) wavefunctions, which are written as a sum of products of one-electron orbitals

$$
\begin{aligned}
& |i\rangle=\sum_{l} c_{l}\left|i_{1}^{l}\right\rangle\left|i_{2}^{l}\right\rangle, \\
& |f\rangle=\sum_{j} d_{j}\left|f_{1}^{\prime j}\right\rangle\left|f_{2}^{\prime j}\right\rangle, \\
& |k\rangle=\sum_{s} b_{s}\left|k_{1}^{s}\right\rangle\left|k_{2}^{s}\right\rangle .
\end{aligned}
$$

We have labeled the final one-electron states with prime, meaning that the corresponding oneelectron wavefunctions are calculated with the other electron in the final state. Introducing the initial and final-state CI wavefunctions into the first-order amplitude (2), one gets a sum of products of overlap integrals and one-electron transition amplitudes 


$$
\begin{aligned}
& a^{(1)}= \\
& -i \sum_{l} \sum_{j} c_{l} d_{j}^{*}\left\langle f_{2}^{\prime j} \mid i_{2}^{l}\right\rangle \int_{-\infty}^{+\infty} \mathrm{d} t \mathrm{e}^{\mathrm{i}\left(E_{f}-E_{i}\right) t}\left\langle f_{1}^{\prime j}\left|V_{1}(t)\right| i_{1}^{l}\right\rangle \\
& -i \sum_{l} \sum_{j} c_{l} d_{j}^{*}\left\langle f_{1}^{\prime j} \mid i_{1}^{l}\right\rangle \int_{-\infty}^{+\infty} \mathrm{d} t \mathrm{e}^{\mathrm{i}\left(E_{f}-E_{i}\right) t}\left\langle f_{2}^{\prime j}\left|V_{2}(t)\right| i_{2}^{l}\right\rangle .
\end{aligned}
$$

These terms can be interpreted as follows. The term containing the basic configurations both from the initial and final states $\left(l=1\right.$ and $j=1, c_{1}$ and $d_{1}$ being the largest coefficients), can be regarded as the shake term. If the one-electron orbitals from the initial and the final basic configurations have the same symmetry, the overlap integral $\left\langle f_{i}^{\prime 1} \mid i_{i}^{1}\right\rangle$ (for $i$ equals 1 or 2) may be nonzero, and the shake process contributes to the transition of the second electron.

The terms with $j=1$ and $l \neq 1$ are responsible for the initial-state correlation, while those with $j \neq 1$ and $l=1$ express the final-state correlation. The terms with $j \neq 1$ and $l \neq 1$ contains both initial and final-state correlation, but usually are less important, because both coefficients $c_{l}$ and $d_{j}$ are small.

Using the CI (4) wavefunctions for the secondorder amplitude (3), one obtains

$$
\begin{aligned}
a^{(2)}=- & \sum_{k} \sum_{j, l} \sum_{r, s} d_{j}^{*} c_{l} b_{r} b_{s}^{*}\left\langle f_{2}^{\prime j} \mid k_{2}^{r}\right\rangle\left\langle k_{1}^{s} \mid i_{1}^{l}\right\rangle \\
& \times \int_{-\infty}^{+\infty} \mathrm{d} t \mathrm{e}^{\mathrm{i}\left(E_{f}-E_{k}\right) t}\left\langle f_{1}^{\prime j}\left|V_{1}(t)\right| k_{1}^{r}\right\rangle \\
& \times \int_{-\infty}^{t} \mathrm{~d} t^{\prime} \mathrm{e}^{\mathrm{i}\left(E_{k}-E_{i}\right) t^{\prime}}\left\langle k_{2}^{s}\left|V_{2}\left(t^{\prime}\right)\right| i_{2}^{l}\right\rangle \\
- & \sum_{k} \sum_{j, l} \sum_{r, s} d_{j}^{*} c_{l} b_{r} b_{s}^{*}\left\langle f_{1}^{\prime j} \mid k_{1}^{r}\right\rangle\left\langle k_{2}^{s} \mid i_{2}^{l}\right\rangle \\
& \times \int_{-\infty}^{+\infty} \mathrm{d} t \mathrm{e}^{\mathrm{i}\left(E_{f}-E_{k}\right) t}\left\langle f_{2}^{\prime j}\left|V_{2}(t)\right| k_{2}^{r}\right\rangle \\
& \times \int_{-\infty}^{t} \mathrm{~d} t^{\prime} \mathrm{e}^{\mathrm{i}\left(E_{k}-E_{i}\right) t^{\prime}}\left\langle k_{1}^{s}\left|V_{1}\left(t^{\prime}\right)\right| i_{1}^{l}\right\rangle .
\end{aligned}
$$

The evaluation of the second-order amplitude in this form is practically impossible, because of the infinite number of intermediate states. As we have discussed in a previous paper [39], the closure approximation (one possible method to eliminate the infinite number of the intermediate states) leads to a second-order amplitude with $90^{\circ}$ out of phase relative to the first-order one. Under these conditions the cross section calculated with formula (1), using $a=a^{(1)}+a^{(2)}$, does not depend on the sign of the projectile charge, and one would lose one of the most interesting effects in the twoelectron transitions.

In order to calculate the second-order amplitude, from the infinite number of the intermediate states we keep only the most important ones. These are assumed to be those reachable from the initial and the final state by a single-electron transition. Simplified, in the considered intermediate states, one of the electrons is in its initial state and the other one has reached the final state, with the proper change in the screening. In other words, each of the intermediate states is described by a one-configuration wavefunction, with one of the electrons in an initial, and the other in a final configuration

$|k\rangle=\left|i_{1}^{\prime r}\right\rangle\left|f_{2}^{s}\right\rangle \quad$ or $\quad|k\rangle=\left|i_{2}^{\prime r}\right\rangle\left|f_{1}^{s}\right\rangle$.

The unprimed one-electron states are calculated with the other electron in the initial state, while the primed ones with the other electron in the final state, so the change in the screening (the relaxation of the orbitals [40]) is taken into account. Under these conditions the sum over the intermediate states and intermediate configurations reduces to a sum over the initial and final configurations, and the second-order amplitude becomes

$$
\begin{aligned}
a^{(2)}=- & \sum_{j, r, s, l} d_{j}^{*} c_{l}\left\langle f_{2}^{\prime j} \mid f_{2}^{s}\right\rangle\left\langle i_{1}^{\prime r} \mid i_{1}^{l}\right\rangle \\
& \times \int_{-\infty}^{+\infty} \mathrm{d} t \mathrm{e}^{\mathrm{i}\left(E_{f}-E_{s 2 r 1}\right) t}\left\langle f_{1}^{\prime j}\left|V_{1}(t)\right| i_{1}^{\prime r}\right\rangle \\
& \times \int_{-\infty}^{t} \mathrm{~d} t^{\prime} \mathrm{e}^{\mathrm{i}\left(E_{s 2 r 1}-E_{i}\right) t^{\prime}}\left\langle f_{2}^{s}\left|V_{2}\left(t^{\prime}\right)\right| i_{2}^{l}\right\rangle \\
- & \sum_{j, r, s, l} d_{j}^{*} c_{l}\left\langle f_{1}^{\prime j} \mid f_{1}^{s}\right\rangle\left\langle i_{2}^{\prime r} \mid i_{2}^{l}\right\rangle \\
& \times \int_{-\infty}^{+\infty} \mathrm{d} t \mathrm{e}^{\mathrm{i}\left(E_{f}-E_{s l r 2}\right) t}\left\langle f_{2}^{\prime j}\left|V_{2}(t)\right| i_{2}^{\prime r}\right\rangle \\
& \times \int_{-\infty}^{t} \mathrm{~d} t^{\prime} \mathrm{e}^{\mathrm{i}\left(E_{s 1 r 2}-E_{i}\right) t^{\prime}}\left\langle f_{1}^{s}\left|V_{1}\left(t^{\prime}\right)\right| i_{1}^{l}\right\rangle .
\end{aligned}
$$


Here $E_{s 2 r 1}$ stands for the energy of the intermediate state when one electron is in the $\left|i_{1}^{\prime r}\right\rangle$ state and the other one in the $\left|f_{2}^{s}\right\rangle$, while $E_{s 1 r 2}$ represents the energy of the intermediate state described by the $\left|f_{1}^{s}\right\rangle\left|i_{2}^{\prime r}\right\rangle$ configuration. Because of the different screening $\left\langle i_{1,2}^{\prime r} \mid i_{1,2}^{l}\right\rangle$ does not reduce exactly to $\delta_{r l}$, but usually can be taken so with a very good approximation. The situation is different for the final states, where the change in the screening affects strongly the wavefunctions and the overlap integrals.

In this approximation of the second-order amplitude, electron correlation in the intermediate state is neglected. The effect of the correlation in this case is assumed to be small because of the following. (1) In the case when one electron is in the ground state and the other one in an exited state, the electron-electron interactions can be well described by a screening potential created by the inner electron, and correlation is less important than in the case when both electrons are on the same shell. (2) In the second-order amplitude two projectile-electron interactions are involved, causing the two-electron transition alone; electron correlation in this case leads only to a small correction to the amplitude (as small as the square of the CI coefficients of the neglected configurations are relative to the basic configuration). This was not the case for the first-order amplitude, where only electron-electron interaction can cause the transition of the second electron. The negligible effect of the intermediate-state correlation for high projectile energies is also discussed by Bronk et al. [15].

The above expression of the second-order amplitude (8) takes into account the dependence of the intermediate-state energy of the order of the two projectile-electron interactions, and so does not neglect the time-ordering effect, as the closure approximation [39]. From this fact, it follows that interference between the first-order and the second-order amplitudes is possible, leading to the dependence of the cross section on the sign of the projectile charge.

\section{Results and discussion}

The difficulty in applying the described method for certain two-electron transitions consists in finding good, but relatively simple correlated twoelectron wavefunctions for the initial and the final states. Up to now we have fulfilled this requirement only in case of the double excitation.

When calculating the cross section for the double ionization of the helium, we have applied the Hartree-Fock approximation: electron-electron interactions in the initial, intermediate and final states are taken into account only by a central screening potential, correlation effects have been neglected. Under these conditions we obtain the cross section for antiproton projectiles in the energy range $0.3-2 \mathrm{MeV}$ only $5 \%$ higher than those for protons, instead of the experimental factor-oftwo difference $[38,39]$. Our results for antiprotons are in very good agreement with the experimental data and with those calculated by Ford and Reading [14], but cross sections for protons are too high. This finding is consistent with the discussion of Bronk et al. about the intermediate-state correlation [15]. They state that correlation plays a much more important role in collisions with protons, than with antiprotons, because the proton pulls the electrons toward itself and each other, and the antiproton pushes them away. As can be deduced from our results for the double ionization of the helium, correlation can be neglected even in the initial and the final states, but only for negative projectiles.

We have made calculations with similar uncorrelated wavefunctions for the simultaneous ionization and excitation of the helium [22], obtaining similarly too small differences between cross sections obtained for positive and negative projectiles. We have assumed, that taking into account correlation in the initial state, we could significantly correct our results, because in the final state, with one bound electron and the other leaving the atom, correlation should be unimportant. However, this assumption turned out to be wrong. The inclusion of the initial-state correlation in the calculations does not improve the previous results. Our calculated cross sections for the ionization-excitation of the helium to the $2 p$ state by proton and antiproton impact are plotted in Fig. 1. None of the theoretical calculations fit the experimental data, but ours are the only ones trying to deal with the dependence on the sign of the 


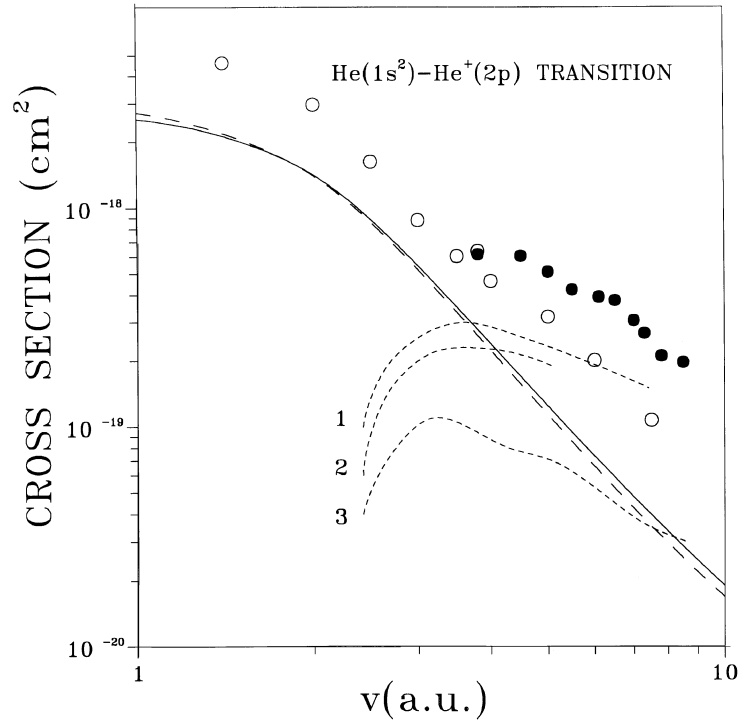

Fig. 1. Cross sections for the ionization-excitation of the helium to the $2 p$ state as a function of the projectile velocity. Solid line represent our results for antiprotons and long dashed line for protons. Short-dashed lines stand for other theoretical cross sections for electron projectiles (1 - Ref. [19], 2 - Ref. [20], 3 Ref. [21]), while full and open circles for experimental data obtained with electron and proton projectiles, respectively [28].

projectile charge, the other theories being only for electrons. In order to improve these calculations, correlation in the final and may be in the intermediate states should be taken into account.

In case of double excitation we have taken into account both initial and final-state correlation. Unfortunately, because of the difficulties in extracting cross sections from the experimental spectra, we have only one reliable data point to compare our results with, that of Moretto-Capelle et al. [37]. Our results for the excitation of the $(2 s 2 p)^{1} \mathrm{P}$ state have been published earlier [34]. As Fig. 2 shows, we do obtain higher cross sections for antiprotons than for protons by $10-20 \%$ in the impact energy range between $100 \mathrm{keV}$ and 10 $\mathrm{MeV}$. Our data are in reasonable accordance to the coupled channel calculation of Fritsch and Lin [30], and in excellent agreement with the recent experimental cross sections of Moretto-Capelle et al. [37] for protons.

The largest difference in cross sections for proton and antiproton impact, up to a factor of 3 ,

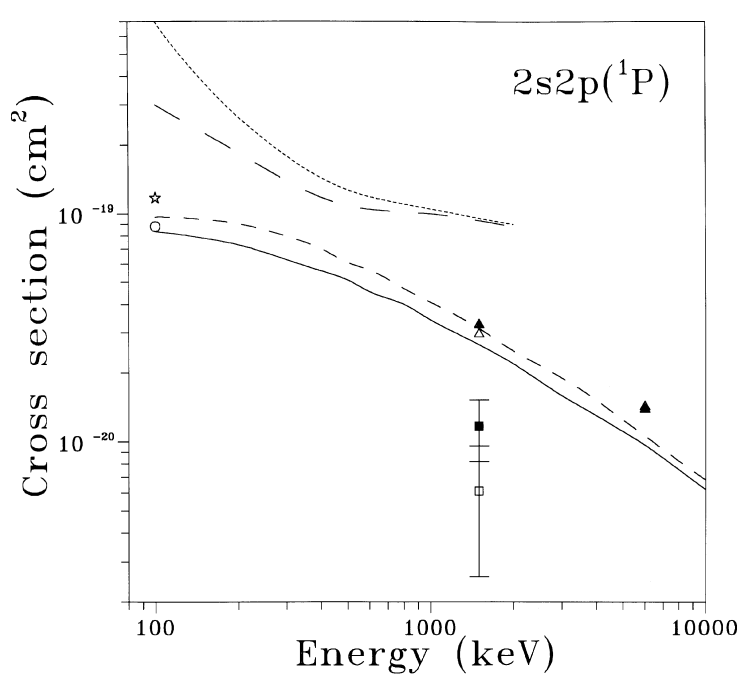

Fig. 2. Cross sections for the double excitation of the helium to the $(2 \mathrm{~s} 2 \mathrm{p})^{1} \mathrm{P}$ state as a function of the projectile energy by proton and antiproton (or equivelocity electron) impact. Our calculated cross sections (solid line: $\mathrm{p}^{+}$, short-dashed line: $\mathrm{p}^{-}$) are compared with the theoretical results of Straton et al. [33] (dotted line: $\mathrm{p}^{+}$, long-dashed line $\mathrm{p}^{-}$), of Fritsch and Lin [30] (triangles) and with the experiments of Giese et al. [36] (squares). Experimental data of Moretto-Capelle et al. [37] are represented by a circle and their theoretical result by a star. Open symbols stand for proton impact, while closed symbols for antiproton (equivelocity electron, in case of the experiments) impact.

have been obtained for the excitation of the $\left(2 \mathrm{~s}^{2}\right)^{1} \mathrm{~S}$ state (Fig. 3) [41]. In this case the first-order amplitude is purely imaginary. The second-order amplitude has a real, non-time-ordered part, and an imaginary part due to the time ordering [39]. The non-time-ordered part roughly means two independent one-electron transitions from $1 \mathrm{~s}$ to $2 \mathrm{~s}$. Because this is not a dipole transition, this part of the amplitude is not large, and the time-ordering (imaginary) part becomes very important. The latter gives with the first-order amplitude a large interference term in the transition probability proportional to $Z^{3}$ ( $Z$ standing for the charge of the projectile), leading to a large difference in cross sections for protons and antiprotons. This conclusion disagree with the theoretical data of Fritsch and Lin [30] and Straton et al. [33], but is in accordance with the theoretical description of the double excitation by Godunov et al. including the 


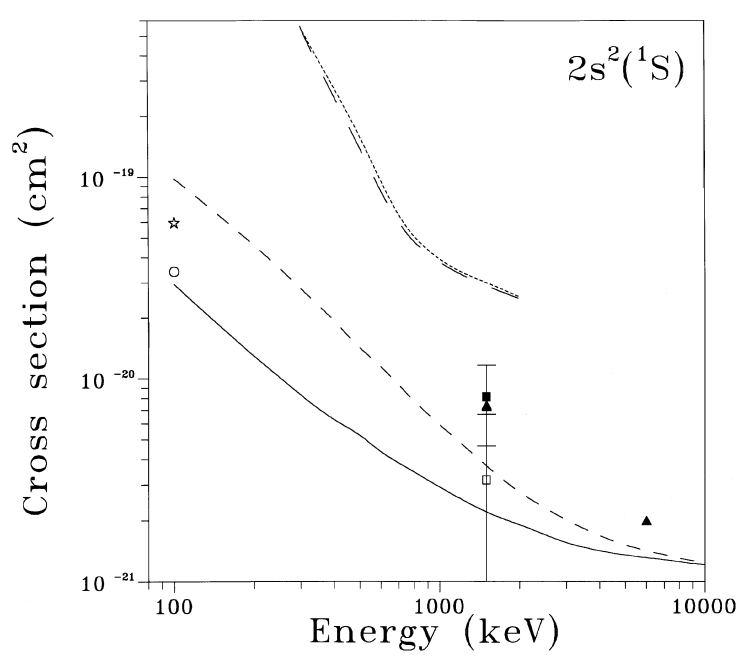

Fig. 3. Same as Fig. 2 but for the excitation of the $\left(2 s^{2}\right)^{1} S$ state.

"excitation via the adjacent electron continuum" [42], and confirms the experimental data of Giese et al. [36]. Again, very good agreement has been found with the $100 \mathrm{keV}$ proton data of MorettoCapelle et al. [37].

The results for the excitation of the $\left(2 \mathrm{p}^{2}\right)^{1} \mathrm{D}$ state [41] are plotted in Fig. 4. The agreement with the recent experimental data of Moretto-Capelle et al. [37] is excellent in this case, too. Here we

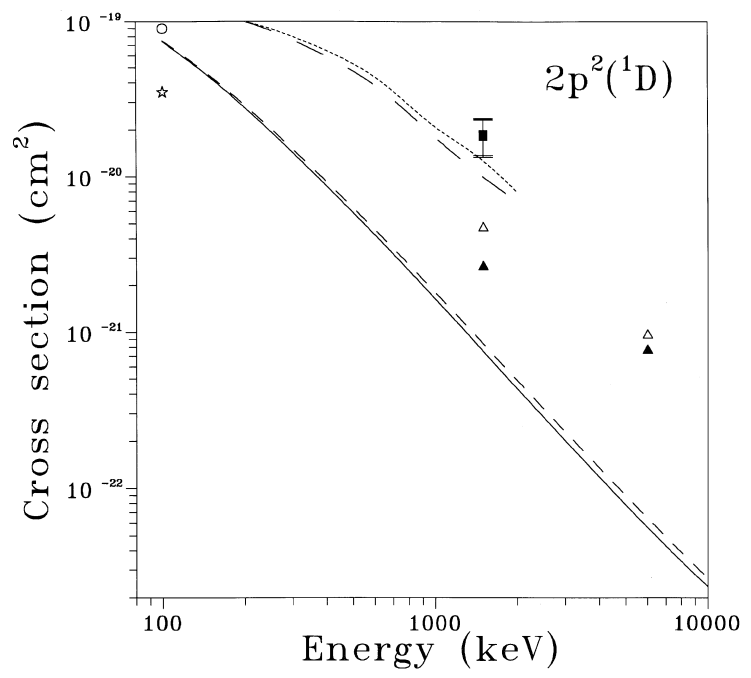

Fig. 4. Same as Fig. 2 but for the excitation of the $\left(2 \mathrm{p}^{2}\right)^{1} \mathrm{D}$ state. have found only weak dependence of the cross section on the sign of the projectile charge. This is due to the fact that the non-time-ordered part of the second-order transition amplitude is a product of two dipole one-electron amplitudes, and dominates above the time-ordering part. Since only the time-ordering part interferes with the first-order amplitude, the interference term in the transition probability proportional to $Z^{3}$ will be small. This conclusion is in agreement with the experimental data of Giese et al. [36], but disagrees with the theoretical analysis of Godunov et al. [42]. The latter have found much larger cross sections for antiprotons than for protons.

\section{Conclusions}

The presented perturbative method is suitable for the calculation of the cross sections for different two-electron transitions induced by charged particles of intermediate and high energies. In order to obtain results in accordance with the experimental data one has to take into account the initial and final-state correlation. We have included these correlation effects in our calculation for the double excitation of the helium, but have neglected the final-state correlation for the double ionization and ionization-excitation. This is the reason why our calculations for the last two transitions do not reproduce the experimental data.

Our results for the double excitation of the helium are in very good agreement with the experimental data of Moretto-Capelle et al. [37], but in order to make definite conclusions upon our data, further experimental investigation is needed.

\section{References}

[1] J.H. McGuire, Electron Correlation Dynamics in Atomic Collisions, Cambridge University Press, Cambridge, 1997.

[2] H.K. Haugen, L.H. Andersen, P. Hvelplund, H. Knudsen, Phys. Rev. A 26 (1982) 1962.

[3] P. Nagy, A. Skutlartz, V. Schmidt, J. Phys. B 13 (1980) 1249.

[4] K. Stephan, H. Helm, T.D. Märk, J. Chem. Phys. 73 (1980) 3763. 
[5] H. Knudsen, L.H. Andersen, P. Hvelplund, G. Astner, H. Cederquist, H. Danared, L. Liljeby, K.G. Rensfelt, J. Phys. B 17 (1984) 3545.

[6] M.B. Shah, H.B. Gilbody, J. Phys. B 18 (1985) 899.

[7] L.H. Andersen, P. Hvelplund, H. Knudsen, S.P. Møller, A.H. Sørensen, K. Elsner, K.G. Rensfelt, E. Uggerhøj, Phys. Rev. A 36 (1987) 3612.

[8] L.H. Andersen, P. Hvelplund, H. Knudsen, S.P. Møller, J.O.P. Pedersen, S. Tang-Petersen, E. Uggerhøj, K. Elsner, E. Morenzoni, Phys. Rev. A 40 (1989) 7366.

[9] P. Hvelplund, H. Knudsen, U. Mikkelsen, E. Morenzoni, S.P. Møller, E. Uggerhøj, T. Worm, J. Phys. B 27 (1994) 925.

[10] J.H. McGuire, Phys. Rev. Lett. 49 (1982) 1153.

[11] R.E. Olson, Phys. Rev. A 36 (1987) 1519.

[12] L. Végh, Phys. Rev. A 37 (1988) 992.

[13] J.F. Reading, A.L. Ford, Phys. Rev. Lett. 58 (1987) 543; J. Phys. B 20 (1987) 374

[14] A.L. Ford, J.F. Reading, J. Phys. B 27 (1994) 4215.

[15] T. Bronk, J.F. Reading, A.L. Ford, J. Phys. B 31 (1998) 2477.

[16] L. Meng, R.E. Olson, R. Dörner, J. Ullrich, H. SchmidtBocking, J. Phys. B 26 (1993) 3387.

[17] D.P. Marshall, C. Le Sech, D.S.F. Crothers, J. Phys. B 26 (1993) L219.

[18] T. Das, F. Bary Malik, J. Phys. B 30 (1997) 1223.

[19] M.R.H. Rudge, J. Phys. B 21 (1988) 1887.

[20] A. Raeker, K. Bartschat, R.H.G. Reid, J. Phys. B 27 (1994) 3129.

[21] A. Franz, P.L. Altick, J. Phys. B 28 (1995) 4639.

[22] L. Nagy, J. Wang, J.C. Straton, J.H. McGuire, Phys. Rev. A 52 (1995) R902.

[23] V.A. Sidorovich, J. Phys. B 30 (1997) 2187.

[24] J.L. Forand, K. Becker, J.W. McConkey, J. Phys. B 18 (1985) 1409.
[25] J.O.P. Pedersen, F. Folkmann, J. Phys. B 23 (1990) 441.

[26] K.-H. Schartner, B. Lommel, D. Detleffsen, J. Phys. B 24 (1991) L13.

[27] S. Fülling, R. Bruch, E.A. Rauscher, P.A. Neil, E. Träbert, P.H. Heckmann, J.H. McGuire, Phys. Rev. Lett. 68 (1992) 3152.

[28] M. Bayley, R. Bruch, E. Rausher, S. Bliman, J. Phys. B 28 (1995) 2655.

[29] A.L. Godunov, V.A. Schipakov, P. Moretto-Capelle, D. Bordenave-Montesquieu, M. Benhenni, A. BordenaveMontesquieu, J. Phys. B 30 (1997) 5451.

[30] W. Fritsch, C.D. Lin, Phys. Rev. A 41 (1990) 4776.

[31] T.G. Winter, Phys. Rev. A 43 (1991) 4727.

[32] K. Moribayashi, K. Hino, M. Matsuzawa, M. Kimura, Phys. Rev. A 44 (1991) 7234.

[33] J.C. Straton, J.H. McGuire, Z. Chen, Phys. Rev. A 46 (1992) 5514.

[34] L. Nagy, D. Bodea, Nucl. Instr. and Meth. B 124 (1997) 401.

[35] J.O.P. Pedersen, P. Hvelplund, Phys. Rev. Lett. 62 (1989) 2373.

[36] J.P. Giese, M. Schultz, J.K. Swenson, H. Schöne, M. Benhenni, S.L. Varghese, C.R. Vane, P.F. Dittner, S.M. Shafroth, S. Datz, Phys. Rev. A 42 (1990) 1231.

[37] P. Moretto-Capelle, D. Bordenave-Montesquieu, A Bordenave-Montesquieu, A.L. Godunov, V.A. Schipakov, Phys. Rev. Lett. 79 (1997) 5230.

[38] L. Nagy, Nucl. Instr. and Meth 124 (1997) 271.

[39] L. Nagy, J.H. McGuire, L. Végh, B. Sulik, N. Stolterfoht, J. Phys. B 30 (1997) 1239.

[40] N. Stolterfoht, Phys. Rev. A 48 (1993) 2980.

[41] D. Bodea, A. Orbán, D. Ristoiu, L. Nagy, J. Phys. B 31 (1998) L745.

[42] A.L. Godunov, J.H. McGuire, V.A. Schipakov, J. Phys. B 30 (1997) 3227. 